\title{
Твердотельные СВч-переключатели
}

\section{Часть 2}

В. Кочемасов, к. т. н. ${ }^{1}$, А. Кирпиченков, к. т. н. ${ }^{2}$

УДК 621.389 | ВАК 05.27 .00

В первой части статьи, опубликованной в предыдущем номере журнала, рассматривались общие сведения о твердотельных СВЧ-переключателях и технологиях, используемых при их создании. Сейчас речь пойдет о модульных переключателях. Их начали выпускать сразу же после изобретения кремниевых pin-диодов и в дальнейшем постоянно совершенствовали. Анализ номенклатуры модульных устройств показывает, что большая их часть реализуется на кремниевых ріп-диодах. При создании модульных переключателей, как правило, используется стандартный набор схемотехнических решений. Однако конструктивно изделия могут существенно отличаться из-за разного количества переключаемых каналов и диапазона рабочих частот. Рассмотрим различные типы модульных переключателей.

\section{МОДУЛЬНЫЕ SРSТ-ПЕРЕКЛЮЧАТЕЛИ}

Номенклатура SPST-переключателей на ріп-диодах, предлагаемых десятками компаний, чрезвычайно широка (табл. 2, рис. 6). Модульные изделия выпускают с коаксиальными соединителями или в исполнении drop-in (в виде РЧ-вставки). Они доступны для широкого диапазона частот и различаются конструктивно, а также достижимыми значениями вносимых потерь, развязок, времени переключения и входной мощности

SPST-переключатели могут быть как отражательными (рис. 7а), так и поглощающими (рис. 7б). Модель CMCS0337 производства компании CMC (см. рис. 7а), предназначенная для работы в широкой полосе частот 2-18ГГц, обеспечивает время переключения 2 нс и развязку 60 дБ, которая может быть увеличена при использовании большего числа параллельно включенныхдиодов. Фильтры высоких частот на входе и выходе переключателя эффективно подавляют видеопросачивание.

герметичная модель поглощающего п переключателя

ООО «Радиокомп», генеральный директop, vkochemasov@radiocomp.ru.

АО «Институт Микроволновых Инте-

гральных Систем» ("Микро-ВИС»), главный конструктор. с интегрированным драйвером HM192 компании Kratos, выполненная по микрополосковой технологии в исполнении drop-in с последовательно-параллельным включением pin-диодов (рис. 76), имеет в диапазоне

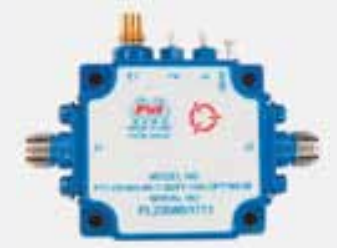

a)

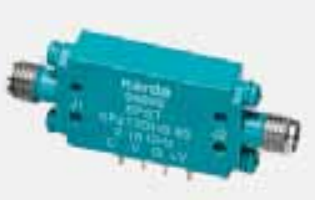

г)

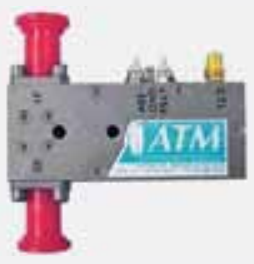

Ж)

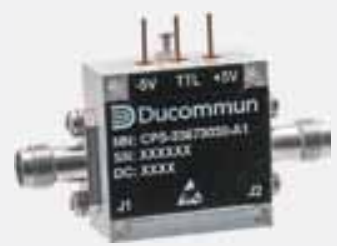

б)

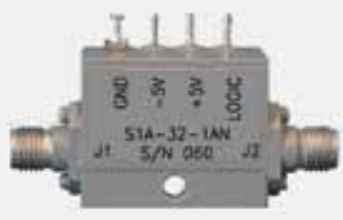

д)

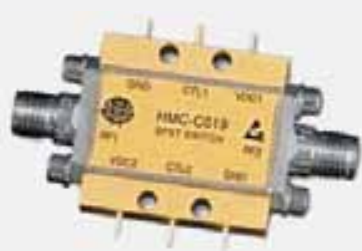

3)

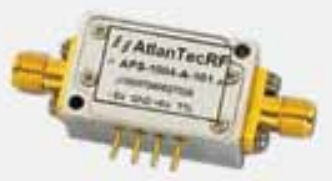

B)

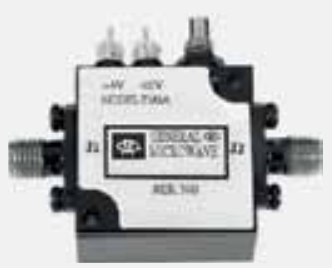

e)

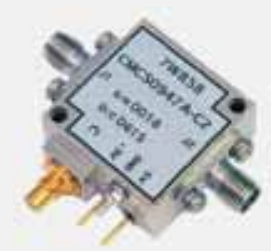

и)
Рис. 6. Конструкции модульных SPST-переключателей: a - P1T-DC40G-65-T-292FF-1NS (PMI); 6 - CPS-33673030-A1 (Ducommun Technologies); в - APS-1004-A-101 (AtlantecRF); г - SP213DHS-80 (Narda Microwave); д - S1A-32-1AN (Mercury Systems); е - F192A (Kratos); ж - S1517D (ATM); з - HMC-C019 (Analog Devices); и - CMCS0947A-C2 (CMC) 
частот 0,2-18,0 ГГц развязку 80 дБ и время переключения $10 \mathrm{HC}$

Амплитудное и фазовое согласование с точностью

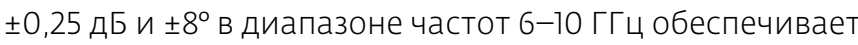
модель CMCS0292 компании CMC, реализованная в герметичном drop-in корпусе, совместимом с микрополосковыми или полосковыми линиями передачи. Типичное время переключения в этом устройстве составляет 0,3 нс.

Около 90 моделей SPST-переключателей отражательного и поглощающего типов выпускает компания Cobham Signal \& Control Solutions. Эти изделия доступны для диапазона частот 0,01-18,00 ГГц. Все они оснащены драйвером, обеспечивающим работу переключателей от источников питания +5 и -12... -18 В. В каждом из устройств на входе и выходе предусмотрена развязка по постоянноMY току.

SPST-переключатели могут быть также реализованы с использованием балансных схем (рис. 8). Одно из таких устройств (модель ISD-9112, компания ST Olektron) в диапазоне частот 0,5-400 МГц обеспечивает вносимые потери 2 дБ, развязку более 45 дБ и время переключения $200 \mathrm{HC}$.

Четыре модели SPST-переключателей производит компания Teledyne Microwave Solutions, в том числе два отражательного (SJ1010, SRS3019) и столько же поглощающего (S2CP20101, S2CP20201) типа. Изделия выпускаются в различном конструктивном исполнении (корпуса ТО-8В, SMTO-8B, SMA и 10-pin Gullwing)

12 моделей отражательного типа и столько же поглощающего (диапазоны частот 0,2-2,0; 0,5-2,0; 2-8; 4-12; 2-18; 1-18 ГГц) предлагает компания Miteq. Время переключения в них не превышает 2 нс. Изделия выпускаются как с SMA-соединителями, так и в исполнении drop-in.

В широком температурном диапазоне $-55 . .95^{\circ} \mathrm{C}$ могут работать SPST-переключатели на ріп-диодах производства компании Mercury Systems. Отражательные

Таблица 2. Характеристики модульных SPST-переключателей

\begin{tabular}{|c|c|c|c|c|c|c|c|}
\hline Компания & Модель & $\begin{array}{r}\text { Диапазон } \\
\text { частот, } \\
\text { ГГц }\end{array}$ & $\begin{array}{l}\text { IL, } \\
\text { дБ }\end{array}$ & $\begin{array}{l}\text { Iso, } \\
\text { дБ }\end{array}$ & $\begin{array}{l}\mathrm{T}_{\mathrm{n}} \\
\mathrm{HC}\end{array}$ & $\mathrm{KCBH}$ & $\begin{array}{r}\mathrm{P}_{\text {доп., }} \\
\mathrm{BT}\end{array}$ \\
\hline $\mathrm{R} \& \mathrm{~K}$ & SW030-0S & $0,005-1,0$ & $<6,0$ & $>35$ & 50 & 1,4 & 0,02 \\
\hline CMC & CMCS0292 & $6,0-10,0$ & $<1,5$ & $>40$ & 0,3 & $<2,0$ & 1,0 \\
\hline CMC & CMCS0337 & $2,0-18,0$ & $<2,2$ & $>50$ & 2 & $<1,9$ & 2,0 \\
\hline Narda Microwave-east & SM213DHS & $2,0-18,0$ & $<2,1$ & $>45$ & 25 & $<1,8$ & 0,5 \\
\hline Narda Microwave-east & SM213D & $2,0-18,0$ & $<2,0$ & $>55$ & 10 & $<1,7$ & 3,0 \\
\hline Magnum Microwave & AHS1802-1xx & $2,0-18,0$ & $\leq 2,4$ & $>60$ & $<25$ & $<1,8$ & - \\
\hline Elbit Systems & MW121T28-H-H & $2,0-18,0$ & $<1,3$ & $>60$ & 15 & $<1,8$ & 0,1 \\
\hline AtlantecRF & APS1006-R & $2,0-18,0$ & 2,2 & 55 & 50 & 2,2 & $0,2-1,0$ \\
\hline Cernex & C1TA02183260Y & $2,0-18,0$ & 3,2 & $>60$ & $<50$ & $<2,2$ & 0,5 \\
\hline Kratos & 9114 & $1,0-18,0$ & $<2,5$ & $>60$ & $<20$ & $<2,0$ & 1,0 \\
\hline MCE & SWM-1100 & $0,5-18,0$ & $<2,75$ & $>50$ & $<100$ & 2,1 & $<0,1$ \\
\hline $\begin{array}{l}\text { Cobham Signal } \\
\text { \& Control Solutions }\end{array}$ & S1U6 & $0,5-18,0$ & $<3,0$ & $>65$ & 100 & $<1,9$ & 0,5 \\
\hline ATM Microwave & S1517D & $0,5-18,0$ & 2,5 & $37-80$ & $<20$ & $<1,8$ & - \\
\hline Miteq & SW1-001200RN1NFS138B & $0,1-20,0$ & $<5,0$ & $>80$ & 35 & $<2,0$ & - \\
\hline MCLI & D1-18 & $18,0-26,5$ & $<3,7$ & 60 & - & $<2,5$ & 0,1 \\
\hline Kratos & F9016 & $0,1-40,0$ & $2,3-5,0$ & $60-30$ & $<250$ & $2,0-2,5$ & $<0,2$ \\
\hline $\mathrm{AMC}$ & SWCH1K-DC40-SK & $0-40,0$ & 5,5 & 65 & 5 & 2,0 & 0,05 \\
\hline Cernex & C1TA18502540S & $18,0-50,0$ & 2,5 & $>40$ & $<100$ & $<1,5$ & 0,25 \\
\hline Pasternack & PE71S2023 & $0,05-67,0$ & $<8,5$ & $>20$ & $<150$ & - & $<0,5$ \\
\hline
\end{tabular}


и поглощающие устройства выпускаются в пяти частотных диапазонах (0,5-2; 2-6; 6-18; 2-18; 0,5-18,0 ГГц). При необходимости их поставляют в исполнениях, устойчивыхк воздействию вибраций, ударов и ускорений.

Широкую линейку отражательных и поглощающих переключателей стандартного типа производит компания Cernex. Самые широкополосные из них, предназначенные для работы в диапазоне частот 33-50 ГГц, обеспечивают вносимые потери от 2,2 дБ, развязку до 40 дБ и время переключения 100 нс.

Герметичные отражательные и поглощающие SPST-переключатели для коммерческого, медицинского, военного и космического применения в диапазонах частот 0,5-4,0; 0,5-8,0; 0,5-12,0; 0,5-18,0; 0,5-20,0 ГГц производит компания AKON. Вносимые потери и время переключения во всех моделях не превышают 1,1-2,4 дБ и 50 нс соответственно. Развязка на всех частотах превосходит 60 дБ.

Коаксиальные SPST-переключатели на ріп-диодах серии QSC компании Quinstar Technology обеспечивают быстрое переключение, высокую развязку и малые вносимые потери в диапазонах частот 18,0-26,5; 26,5-40,0; 18,0-40,0 ГГц, идеально подходят для обеспечения импульсной модуляции и защиты приемников. Доступны как отражательные, так и поглощающие типы этих устройств. Компактный корпус включает в себя драйвер, обеспечивающий время переключения 5 нс.

Исключительно широкополосные (0,05-67,00 ГГц) модели SPST-переключателей отражательного (PE7IS2023) и поглощающего (PE7IS2024) типов выпускаются компанией Pasternack. В этом диапазоне частот устройства имеют время переключения 150 нс и входную мощность до 0,5 Вт. В поглощающих переключателях выходной порт в положении "включено» замыкается на 50-Ом нагрузку. Вносимые потери увеличиваются от 1 дБ на низких частотах до 8-9 дБ на частоте 67 ГГц (рис. 9а, б), развязка по диапазону частот меняется от 20 до 48 дБ в изделии PE7IS2023 и от 25 до 48 дБ - в PE7IS2024 (рис. 9в, г).

Две модели ріп-диодных SPST-переключателей (CPS-33672030-01, CPS-33673030-A1), выполненных по AlGaAs MИC технологии в модульном исполнении с коаксиальными соединителями 1,85 мм, работающих в диапазоне частот 0,05-67 ГГц, предлагает компания Ducommun Technologies. Характеристики этих устройств полностью

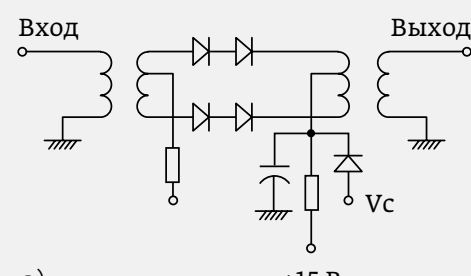

a)

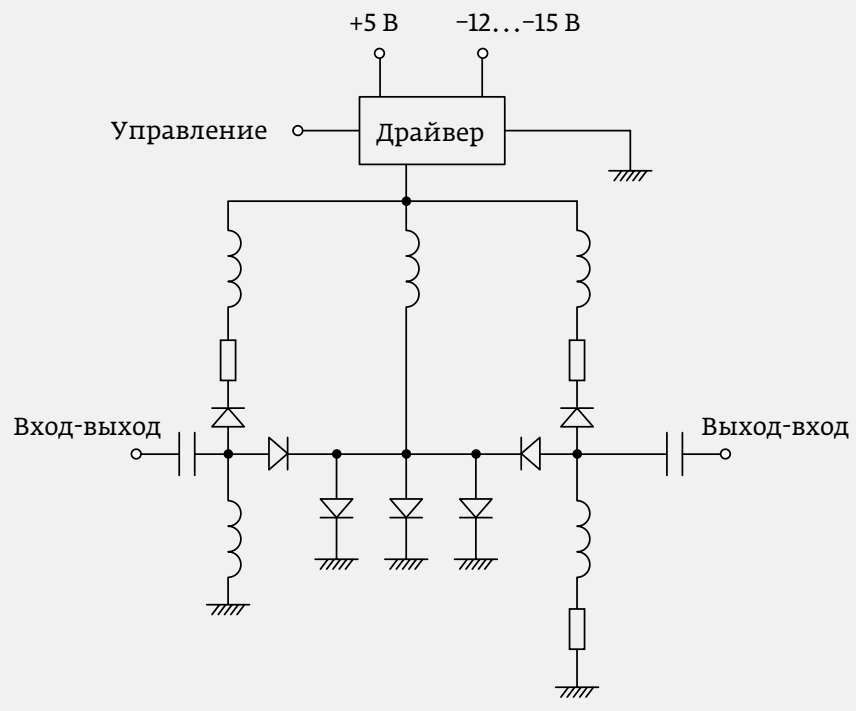

б)

Рис. 7. Принципиальные схемы SPST-переключателей: а - отражательного (модель CMCS0337, компания CMC); б - поглощающего (модель НM192, компания Kratos)

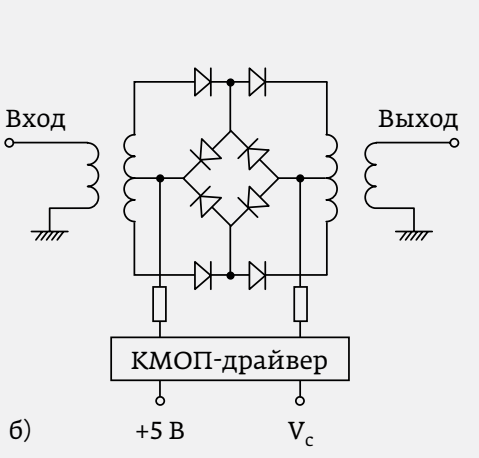

Рис. 8. Балансные схемы SPST-переключателей: а - модель ISD-9112 (ST Olektron); 6 - модель SW030-0S (R\&K) 


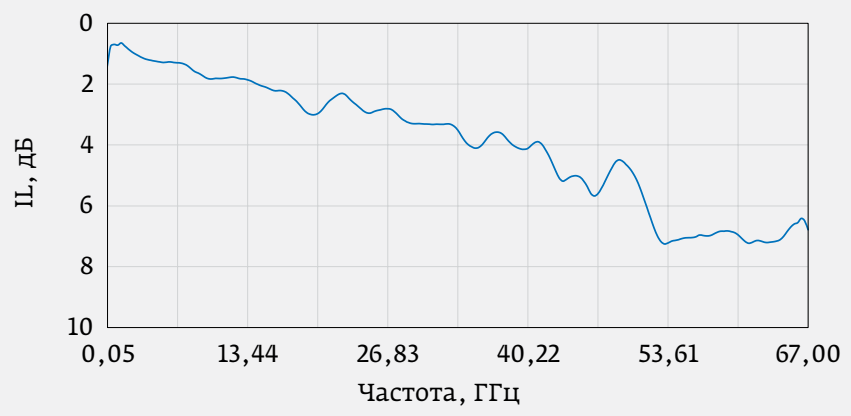

a)

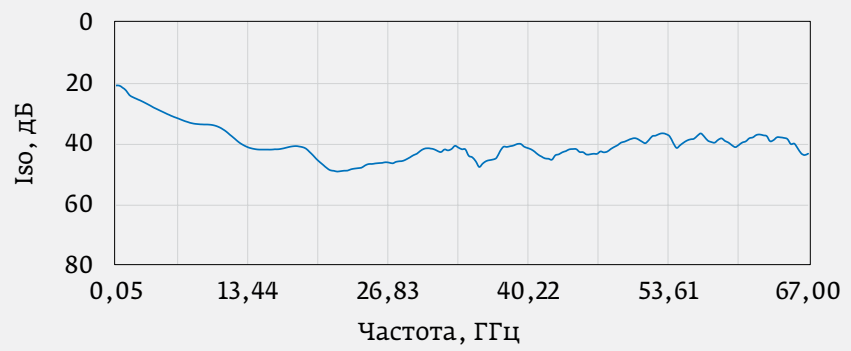

B)

Рис. 9. Зависимости вносимых потерь (а, б) и развязки (в, г) в отражательном PE71S2023 (а, в) и поглощающем PE71S2024 (б, г) переключателях компании Pasternack

совпадают с характеристиками изделий, выпускаемых компанией Pasternack.

Во многих случаях SPST-переключатели используются в качестве импульсных модуляторов. Специально разработанный компанией РМІ импульсный модулятор МРМ-1002 в диапазоне частот 8-18 ГГц формирует радиоимпульсы с подавлением сигнала входной частоты на 61 дБ. Полученные в результате тестовых испытаний времена нарастания, спада и переключения составляют 10,2; 18,5 и 33,4 нс соответственно.

Три серии SPST-переключателей отражательного типа (SFS, DJS и HFS) выпускает компания Sierra Microwave Technology. Устройства SFS-серии представлены шестью

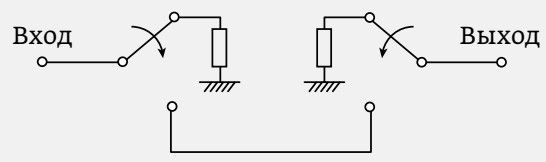

Рис. 10. Схематичное изображение структуры переключателей НMC-C019 и НMC-C583 (компания Analog Devices)

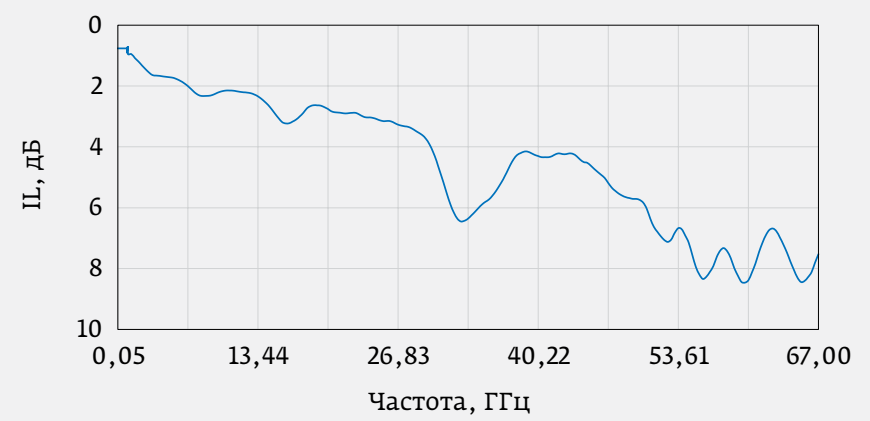

б)

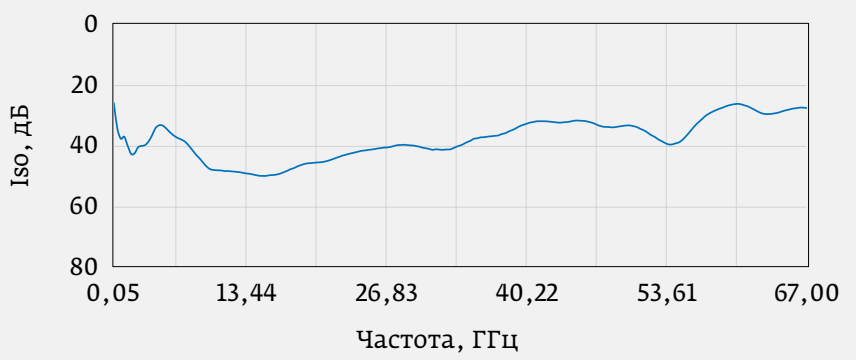

г)

моделями в диапазонах частот 0,5-4,0; 0,5-8,0; 0,5-12,0; 0,5-18,0; 0,5-20,0; 0,5-26,5 ГГц. Вносимые потери по мере увеличения частоты меняются от 1,1 до 3,0 дБ, минимальная развязка составляет 60 дБ, а время переключения 25 нс. Миниатюрная серия DJS в тех же частотных диапазонах, что и SFS-серия, обеспечивает сходные характеристики и может быть реализована как с коаксиальными соединителями, так и в исполнении drop-in. Высокочастотная HFS-серия SPST-переключателей представлена одной моделью HFS1840-OX1 отражательного типа (вносимые потери составляют менее 4,5 дБ, развязка более 50 дБ и скорость переключения 2 мс). Модель выполнена с соединителями К-типа.

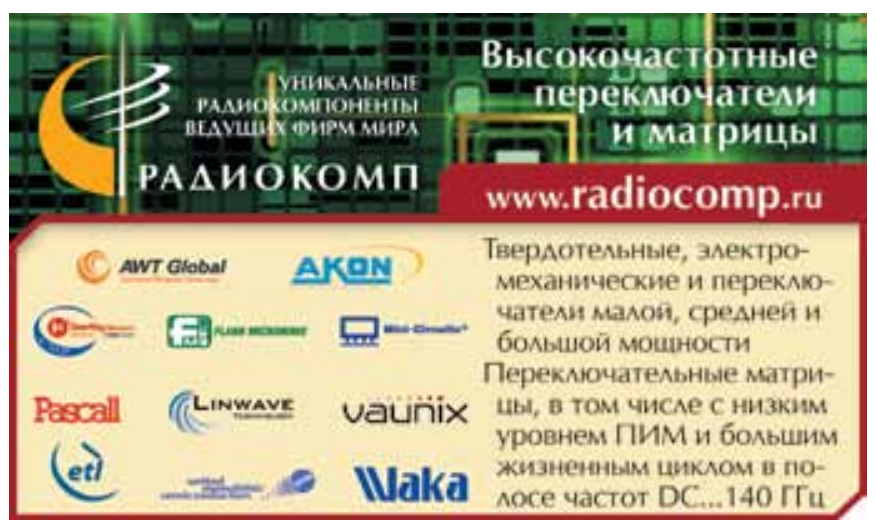


Две герметизированные модели поглощающих SPSTпереключателей (рис. 10), выполненных по технологиям GaAs MESFET и GaAs PHEMT, представляет компания Analog Devices. Первое устройство (HМC-C019), предназначенное для работы в диапазоне частот 0-20 ГГц, обеспечивает вносимые потери менее 7 дБ, развязку 100 дБ на частоте 4 ГГц и 65 дБ на частоте 20 ГГц. Второе устройство (НMC-С583) для диапазона частот 0,1-40 ГГц обеспечивает типовые значения вносимых потерь и развязки 7 и 50 дБ соответственно.

\section{МОДУЛЬНЫЕ SРDТ-ПЕРЕКЛЮЧАТЕЛИ}

Изделия этого типа - одни из наиболее востребованных и весьма разнообразны конструктивно (рис. 11). SPDT-переключатели в модульном исполнении доступны в широком диапазоне частот и удовлетворяют различным требованиям (табл. 3). Благодаря практически неограниченному сроку службы эти ріп-диодные устройства нашли применение в лабораторном и тестовом оборудовании, а также в фазированных антенных решетках, автомобильных и медицинских применениях и др.

Большая часть компаний выпускает переключатели как отражательного, так и поглощающего типов. При этом используются все три конфигурации: последовательная, параллельная и последовательно-параллельная.

В рамках принципиальной схемы поглощающего SPDTпереключателя (рис. 12) возможны различные модификации. Так, увеличивая число параллельно включенных pinдиодов, можно добиться более высокой развязки, а путем включения фильтров высокой частоты по одному, двум или трем портам - снизить видеопросачивание. При исключении из схемы резистора R и диода D, выделенных красным цветом (см. рис. 12), и замыкании между собой точек 1 и 2 переключатель становится отражательным.

Таблица 3. Характеристики модульных SPDT-переключателей

\begin{tabular}{|c|c|c|c|c|c|c|c|}
\hline Компания & Модель & $\begin{array}{r}\text { Диапазон } \\
\text { частот, } \\
\text { ГГц }\end{array}$ & $\begin{array}{l}\text { IL, } \\
\text { ДБ }\end{array}$ & $\begin{array}{l}\text { Iso, } \\
\text { дБ }\end{array}$ & $\begin{array}{l}\mathrm{T}_{\Pi}, \\
\mathrm{HC}\end{array}$ & $\mathrm{KCBH}$ & $\begin{array}{r}\mathrm{P}_{\text {доп, }} \\
\mathrm{BT}\end{array}$ \\
\hline RLC Electronics & SSD-12418 & $12,4-18,0$ & $<3,5$ & $>60$ & 50 & 2,1 & 0,25 \\
\hline Miteq & SW2-120180AI2HS & $12,0-18,0$ & $<2,8$ & $>50$ & $<35$ & $<2,1$ & - \\
\hline AtlantecRF & APS-2006-R & $2,0-18,0$ & 2,4 & 60 & 60 & 2,2 & 0,2 \\
\hline Narda Microwave-East & S2S7R & $2,0-18,0$ & $<2,9$ & $>60$ & $<100$ & $<2,0$ & 0,5 \\
\hline Cernex & C2TA02183260Y & $2,0-18,0$ & 3,2 & $>60$ & $<50$ & $<2,2$ & 0,5 \\
\hline Miteq & SW2-010180RN1NF & $1,0-18,0$ & $<3,0$ & $>70$ & 20 & $<2,1$ & - \\
\hline $\mathrm{MCE} / \mathrm{KDI}$ & SWM-1200 & $0,5-18,0$ & $<2,75$ & $>50$ & $<100$ & $<2,1$ & 0,1 \\
\hline Sage Millimeter & SKD-0521833360-SFSF-A1 & $0,5-18,0$ & 3,6 & 60 & 100 & 2,0 & 0,5 \\
\hline API Technologies & MASWML0012 & $0,5-18,0$ & $<2,6$ & $>55$ & $<30$ & - & $<0,1$ \\
\hline ATM Microwave & S2317D & $0,5-18,0$ & 2,5 & 60 & $<250$ & $<2,0$ & - \\
\hline Keysight & P9402C & $0,1-18,0$ & 4,0 & 80 & 380 & - & 0,2 \\
\hline CMC & SN20-314 & $0,1-20,0$ & $<4,0$ & $>75$ & 500 & $<2,3$ & 1,0 \\
\hline Cernex & C2TR33503530S & $33,0-50,0$ & 3,5 & $>30$ & $<100$ & $<1,5$ & 0,25 \\
\hline Sage Millimeter & SKD-1835033530-2F2F-R1 & $18,0-50,0$ & 3,5 & 30 & 100 & 1,5 & 0,25 \\
\hline PMI & P2T-100M50G-100-T & $0,1-50,0$ & $5,0-9,0$ & $70-100$ & $<50$ & $2,2-3,0$ & $<0,1$ \\
\hline Keysight & $85331 B$ & $0,045-50,0$ & 15,5 & 75 & 1000 & - & 0,5 \\
\hline Pasternack & PE71S2026 & $0,1-67,0$ & $<10,5$ & $>40$ & $<100$ & - & $<0,5$ \\
\hline RF-Lambda & RFSP2TA3080G & $30,0-80,0$ & 3,0 & $>40$ & - & 1,5 & $<0,2$ \\
\hline Cernex & C2TR75853025S & $75,0-85,0$ & 3,0 & $>25$ & $<100$ & $<1,5$ & 0,25 \\
\hline Sage Millimeter & SKD-9031043525-1010-R1 & $90,0-100,0$ & 3,5 & 25 & 100 & 1,5 & 0,25 \\
\hline
\end{tabular}


Характеристики переключателей во многом зависят от идентичности используемых ріп-диодов, на что обращается внимание в особо ответственных изделиях.

чрезвычайно широкую линейку из почти 100 SPDT-переключателей отражательного и поглощающего типа выпускает компания Cobham Signal \& Control Solutions. Во все переключатели этой компании встроены драйверы, обеспечивающие питание +5 и -12...-18 В. Развязка по постоянному току достигается путем включения DC-блоков на входах всех трех портов. Высокая культура тестирования позволяет выпускать эти устройства в индустриальном, военном и космическом исполнении.

Герметичные переключатели для диапазона частот 0,5-18,0 ГГц, которые производит компания Elbit Systems, отличаются малыми вносимыми потерями, высоким значением развязки и низким уровнем видеопросачивания. Стандартные изделия оснащены драйвером. Опционально переключатели могут работать от внешних драйверов. Примерно в таком же диапазоне (2-18 ГГц) поставляются устройства компании LNX. Уровень видеопросачивания в них не превышает -60 дБм. По мере увеличения

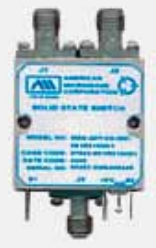

a)

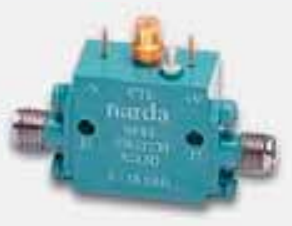

д)

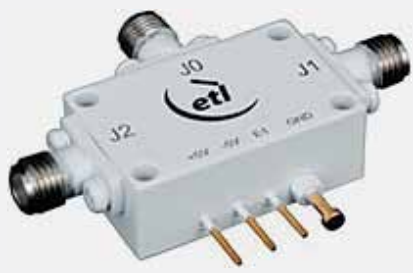

и)

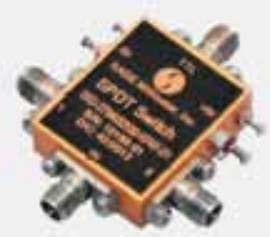

H)

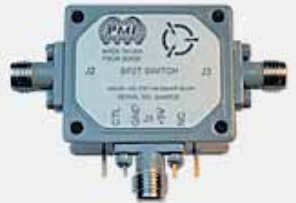

б)

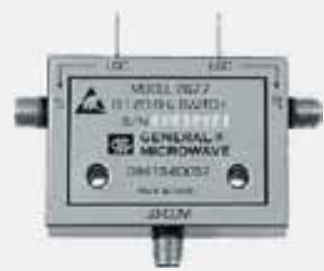

B)

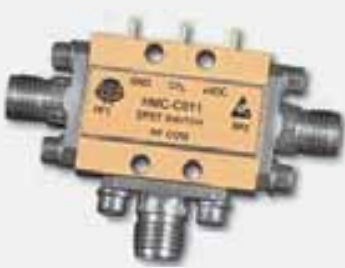

г)

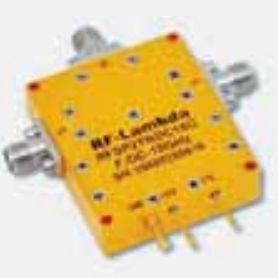

e)

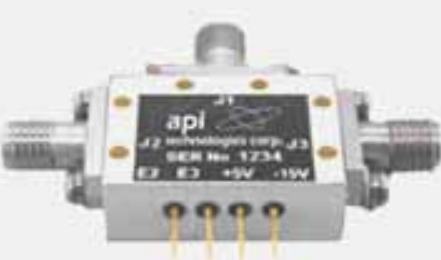

к)

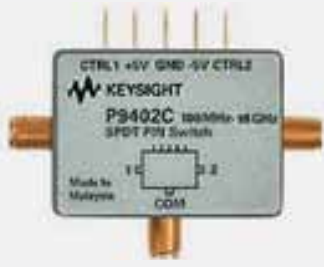

ж)

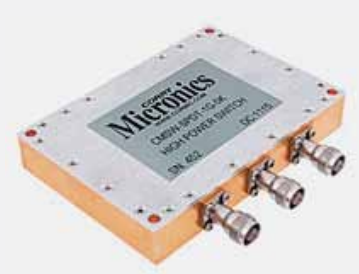

л)

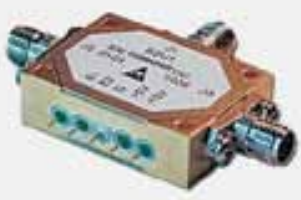

п)

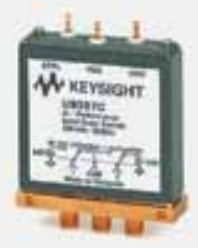

3)

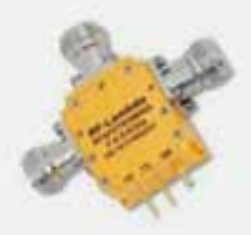

M)

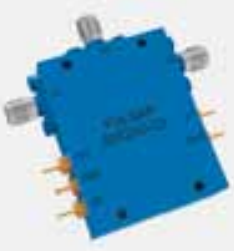

p)

Рис. 11. Конструкции SPDT-переключателей на ріп-диодах: а - MSN-2DT-05-IND (AMC), 6- P2T-100M50G-100-T (PMI), в - 2677 (Kratos), г - HMC-C011 (Analog Devices), д - S123BD (Narda Microwave); e-RFSP2TRDC18C (RF-Lambda); ж-P9402C (Keysight); з - U9397C (Keysight); и - SW-SPDT-KX-1600 (ETL); к - MASWML0012 (API Technologies); л - CMSW-SPDT-1G-5K (Corry Micronics); м-RFSP2TR5M06C (RF-Lambda); н - серия SKD (Sage Millimeter); o - APS-2008-R-202 (AtlantecRF); $\Pi$-S2U1 (Cobham Signal \& Control Solutions); p - SW2AD-23 (Pulsar Microwave) 


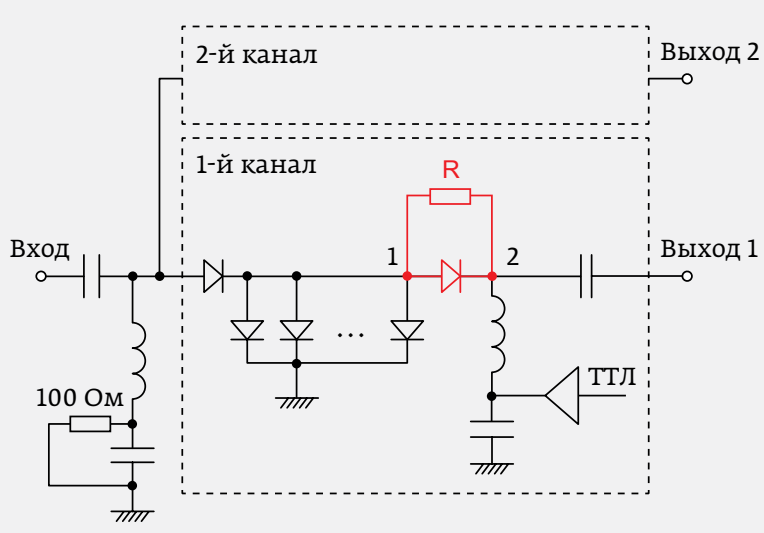

Рис. 12 Принципиальная схема ріп-диодного поглощающего SPDT-переключателя (серия SN20-31x, компания CMC)

верхней границы рабочего диапазона характеристики переключателя обычно ухудшаются.

Переключатели отражательного и поглощающего типов компании API Technologies (модели MASWML0010 и MASWMLO012), выпускаемые в диапазоне частот 0,5-18,0 ГГц, отличаются миниатюрностью, низкими вносимыми потерями (3,3 дБ), хорошей (не менее 55 дБ) развязкой и малым (30 нс) временем переключения, которое обеспечивают ТТЛ-совместимые драйверы. При снятии соединителей эти устройства могут быть подключены к микрополосковым линиям передачи.

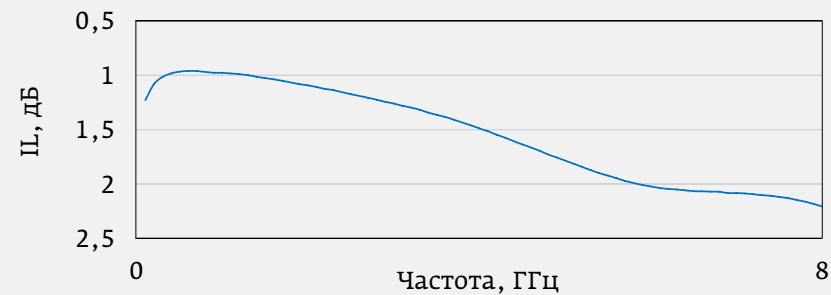

a)

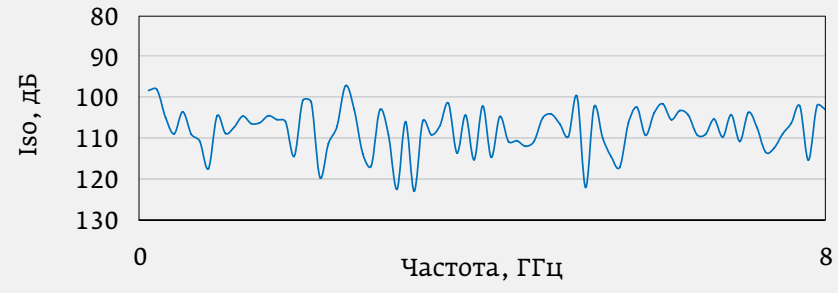

B)

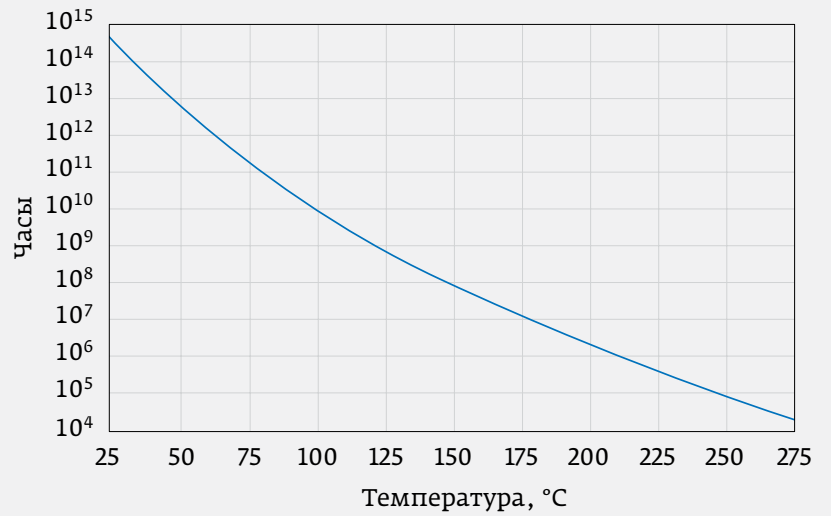

Рис. 13. Средняя наработка на отказ в зависимости от температуры окружающей среды

Многие производители выпускают переключатели в исполнении drop-in в стандартной конфигурации. Примером таких изделий являются модели SW2-120180AN2HS и SW2120180AI2H3 компании Miteq.

Одна из продуктивно работающих на рынке СВЧпереключателей компаний - Cernex - выпускает изделия отражательного и поглощающего типов в диапазоне частот от 0,001 до 100 ГГц. Общее число производимых моделей превышает 50, в том числе в диапазонах 50-60, 55-62, 55-65, 70-86, 75-85 и 90-100 ГГц. Переключатели, рассчитанные на работу до 100 ГГц, производит

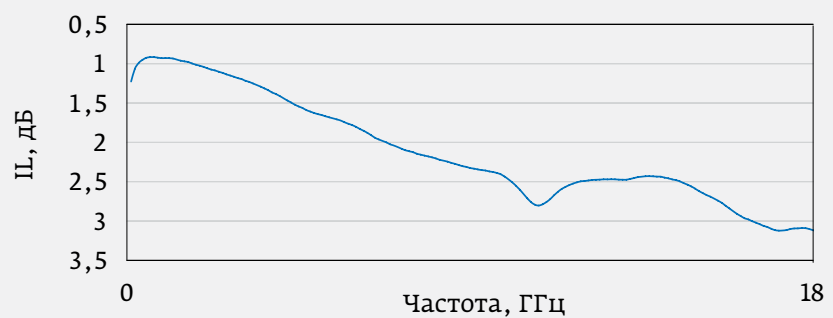

б)

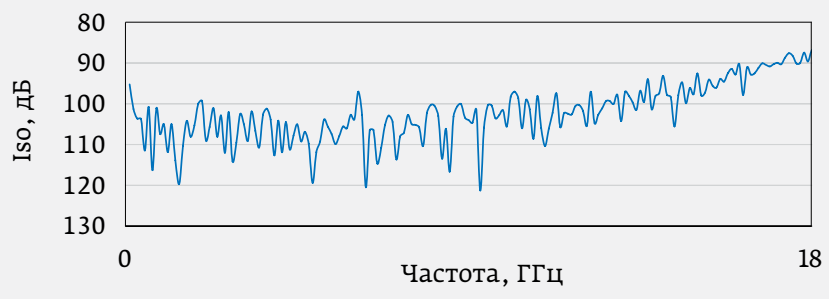

г)

Рис. 14. Зависимости вносимых потерь (а, б) и развязки (в, г) от частоты для переключателей Р9402А (а, в) и Р9402C (б, г) (компания Keysight) 


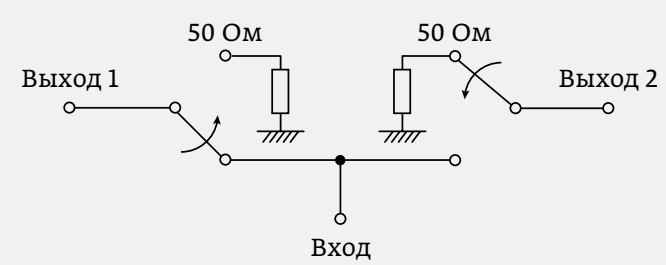

Рис. 15. Упрощенная схема поглощающих переключателей НMC-С011 и НMC-C058 (компания Analog Devices)

также компания Sage Millimeter, причем до 18 ггц изделия могут быть как отражательными, так и поглощающими. Свыше 18 ГГц - в диапазонах 18,0-26, 5; 26, 5-40,0; $18,0-40,0 ; 33,0-50,0 ; 18,0-50,0 ; 45,0-55,0 ; 55,0-65,0$; 75,0-85,0 и 90,0-100,0 ГГц поставляются только изделия отражательного типа.

Широкополосные переключатели в диапазонах частот 0,05-40,0 ГГц и 0,1-50,0 ГГц предлагает компания РМ (модели Р2Т-50M40G-95-T-515-292FF, Р2T-100M50G-100-Т) Особенностью этих изделий является очень высокий уровень развязки, достигающий 100 дБ.

Широкую номенклатуру ріп-диодных переключателей производит компания RF-Lambda. Модель RFSP2TA3080G переключателя отражательного типа для диапазона частот 30-80 ГГц обеспечивает низкие вносимые потери (3 дБ), достаточно высокую (более 45 дБ) развязку и малый КСВН=1,5. Температурный коэффициент,

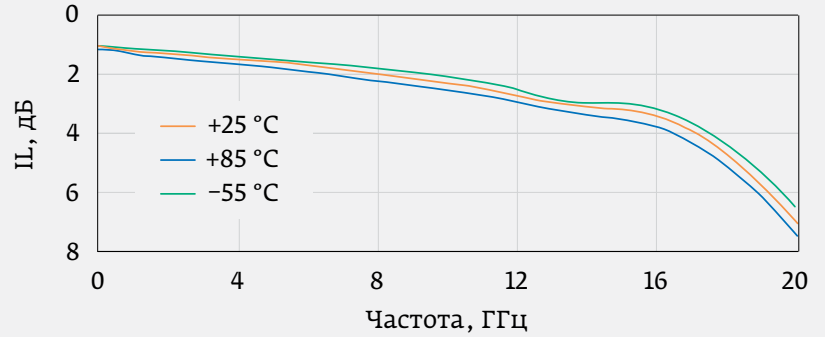

Рис. 16. Зависимости вносимых потерь от частоты в поглощающем переключателе HMC-C058 (Analog Devices) при различных значениях температуры окружающей среды

влияющий на уровень вносимых потерь, не превышает 0,003 дБ/ ${ }^{\circ} \mathrm{C}$. Представляет также интерес график зависимости среднего времени наработки на отказ от температуры (рис. 13).

Исключительно широкополосные ріп-диодные переключатели разработаны компаниями Pasternack (0,1-67,0 ГГц) и Ducommun Technologies (0,05-67,00 ГГц). В качестве высокочастотных в обоих случаях используются соединители 1,85 мм. Управление переключателями осуществляется через SMA-соединители. Каждая компания выпускает по два SPDT-переключателя отражательного и поглощающего типов, соответственно модели PE7IS2025 и PE7IS2026 производства Pasternack и модели

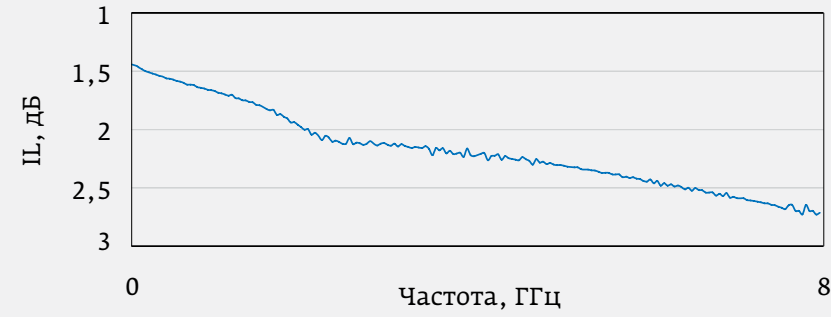

a)

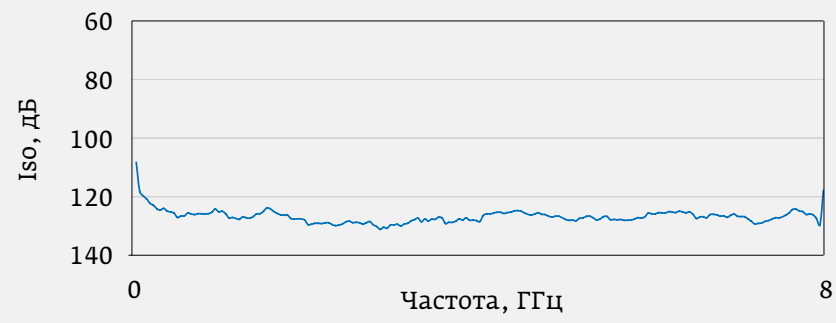

B)

Рис. 17. Зависимости вносимых потерь (а, б) и развязки (в, г) от частоты для SPDT-переключателей U9397A (а, в) и U9397C (б, г), выпускаемых компанией Keysight

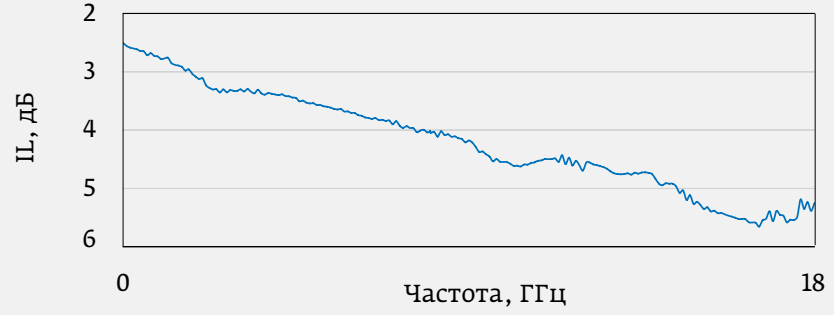

6)

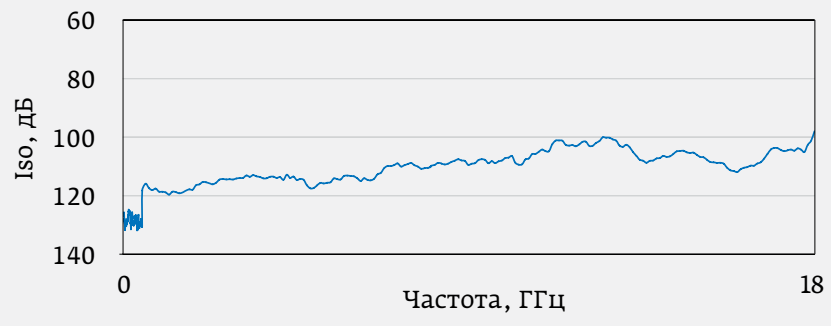

г) 
CPD-33673030-01 и CPD-33674060 компании Ducommun Technologies.

В некоторых SPDT-переключателях предусмотрено согласование амплитудных и фазовых характеристик (между входным и выходными портами). Так, в модели F9321т компании Kratos точность поддержания амплитудных и фазовых характеристик одинаковыми составляет 1 дБ и $12^{\circ}$ соответственно. Одной из мер, способствующих идентичности этих характеристик, является симметричное относительно входного соединителя расположение выходных соединителей на одной из боковых граней.

Два ріп-диодных переключателя Р9402А и P9402С разработаны компанией Keysight для применения в диапазонах частот 0,1-8,0 и 0,1-18,0 ГГц. Особенность этих изделий заключается в возможности их эксплуатации в широком диапазоне температур $\left(-55 \ldots 95^{\circ} \mathrm{C}\right)$, в условиях повышенной влажности, при наличии ударныхи вибрационных нагрузок, на высоте до 15300 м, а также при сильных электростатических воздействиях. Эти ріп-диодные поглощающие переключатели обеспечивают низкие значения вносимых потерь и высокие значения развязки (рис. 14) во всем диапазоне рабочих частот. Оба изделия оснащены ріп-диодным интегральным SPDT-переключателем, дополненным дискретными шунтирующими ріп-диодами в каждом из двух каналов. Введение дополнительных шунтирующих ріп-диодов обеспечивает увеличение развязки между портами. В отличие от других SPDT-переключателей, эти две модели предусматривают три возможных состояния: подключение первого или второго каналов, блокирование прохождения входного сигнала на первый и второй выходы. Достигнутый уровень развязки (рис. 14) ставит эти переключатели в один ряд с электромеханическими изделиями.

В модульных решениях могут также использоваться переключатели, выполненные по другим технологиям. Так, в двух поглощающих переключателях HMC-C011 и HMC-C058 (рис. 15) применяется технология GaAs MESFET, обеспечивающая работу в диапазонах 0-20 и 0-18 ГГц соответственно. Во втором из этих переключателей уровень вносимых потерь меняется по диапазону от 1,2 до 7,0 дБ, а развязка находится в пределах 85-47 дБ. Следует отметить малую зависимость данных показателей от температуры (рис. 16). Оба переключателя выполнены в герметичном исполнении.

При тестировании мобильных телефонов и базовых станций, в системах встроенного контроля, а также в МІмО-применениях могут быть использованы переключатели компании RANATEC, изготовленные по технологии печатных плат. Выпускаемая компанией модель RI 2421 оснащена тремя SPDT-переключателями, работающими в диапазоне частот 0-6 ГГц при вносимых потерях 1,0-1,3 дБ и развязке 55-40 дБ. Время переключения в этой модели не превышает 10 мкс. Подключение к внешним устройствам осуществляется через SMA-соединители.

Чрезвычайно высокий уровень характеристик (рис. 17) обеспечивают выполненные по FET-технологии переключатели U9397А и U9397С компании Keysight. Следует отметить достигнутый в переключателе U9397А уровень развязки 120 дБ во всем диапазоне рабочих частот.

Продолжение следует.

\section{КНИГИ ИЗДАТЕЛЬСТВА «ТЕХНОСФЕРА»}

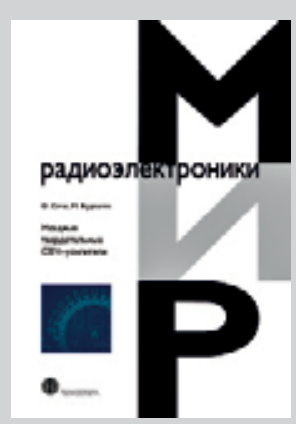

Цена 975 руб.

\section{МОЩНЫЕ ТВЕРДОТЕЛЬНЫЕ СВЧ-УСИЛИТЕЛИ} Сечи Ф., Буджатти М. При поддержке АО "НПП „Исток" им. Шокина" Перевод с англ. nод ред. д. т. н. А. А. Борисова

В книге рассмотрены все традиционные вопросы, связанные с разработкой усилителей мощности, начиная от получения моделей приборов на большом сигнале и заканчивая обсуждением сумматоров мощности и методов проектирования.

Книга представляет интерес для специалистов, которые занимаются разработкой усилителей мощности для базовых станций сотовой связи. В особенности это относится к рассмотрению моделей на больших сигналах, проблем, связанных с фазовыми шумами, методов проектирования усилителей мощности, специальных конструкций усилителей мощности и теплового проектирования. Также данная книга может послужить в качестве справочного пособия при углубленном изучении СВЧ-устройств.

КАК ЗАКАЗАТЬ НАШИ КНИГИ?

$\bowtie$ 125319, Москва, а/я 91; + +7 495 234-0110; 8 +7 495 956-3346; knigi@technosphera.ru, sales@technosphera.ru 